Supporting Information for

\title{
Enzymatically Synthesized DNA Polymer as Co-Carrier for Enhanced RNA Interference
}

Jiantao Yu ${ }^{l, 2}$, Jing Li ${ }^{1}$, Shiyao Zhai ${ }^{1}$, Li Lin ${ }^{1}$, Kui Wang ${ }^{3}$, Bin Tang ${ }^{3}$, Hong Meng ${ }^{2}$, and Leilei Tian ${ }^{l *}$

${ }^{1}$ Department of Materials Science and Engineering, Southern University of Science and Technology, 1088 Xueyuan Blvd., Nanshan District, Shenzhen, Guangdong 518055, P. R. China.

${ }^{2}$ School of Advanced Materials, Peking University Shenzhen Graduate School, Peking University, Shenzhen 518055, China.

${ }^{3}$ Department of Biomedical Engineering, Southern University of Science and Technology, 1088 Xueyuan Blvd., Shenzhen, Guangdong 518055, P. R. China.

*Email: tianll@sustech.edu.cn 


\section{Contains}

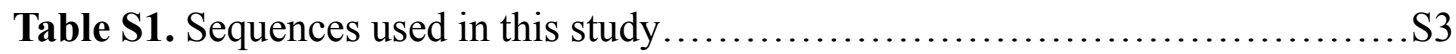

Figure S1. The agarose gel electrophoresis analysis of thermal cleaved RCA chains by heating for different minutes

Figure S2. The agarose gel electrophoresis analysis of the complexation of the RCA6siRNA and RCA 12 -siRNA in the presence of PEI. S5

Figure S3. Polyacrylamide gel electrophoresis analysis of $\mathrm{PEI}_{\mathrm{y}}-\mathrm{RCA}_{18}$-siRNA incubated with DNase I for $30 \mathrm{~min}$. S6

Figure S4. Stability of PEIy-siRNA incubated with/without RNase A for 30 min.... S7 Figure S5. Stability of PEI $\mathrm{I}_{\mathrm{y}}$-siRNA incubated with/without 10\% (a), 20\% (b), 40\% (c), and $50 \%$ (d) of FBS for $12 \mathrm{~h}$. S8

Figure S6. The dissociation property of $\mathrm{PEI}_{y}-\mathrm{RCA}_{18}$-siRNA under the presence of heparin S9

Figure S7. The dissociation property of PEI 10 -siRNA under the presence of heparin $\mathrm{S} 10$

Figure S8. Cell viability of A549-luc cells incubated with of two kinds of polyplexes, $\mathrm{PEI}_{\mathrm{y}}$-siRNA and $\mathrm{PEI}_{\mathrm{y}}-\mathrm{RCA}_{18}$-siRNA (siRNA amount equivalent to 5 pmol for all the polyplexes) after a 12-h incubation. S1 1 
Table S1. Sequences used in this study.

\begin{tabular}{|c|c|}
\hline Sequence Name & \\
\hline RCA Template & $\begin{array}{l}5^{\prime} \text {-ACT TCA CCC TAA CTC TGC CTC CAC TCA } \\
\text { CTT TCA CTC TTG TTC ATC TTC CAC ACT CCA } \\
\text { CTC CCT ACT CG-3' }\end{array}$ \\
\hline RCA Primer & 5'-phosphate- GTG AAG TCG AGT AG-3' \\
\hline siRNA-antisense strand & $\begin{array}{l}5^{\prime}-r U r U r U \text { rGrUrA rUrUrC rArGrC rCrCrA } \\
\text { rUrArG rCdTdT-3' }\end{array}$ \\
\hline siRNA-sense strand & $\begin{array}{l}5^{\prime}-\text { rGrCrU rArUrG rGrGrC rUrGrA rArUrA } \\
\text { rCrArA rAdTdT-3' }\end{array}$ \\
\hline DNA6-sense strand & $\begin{array}{l}5^{\prime}-\mathrm{dTdCdC} \text { dAdCdT rGrCrU rArUrG rGrGrC } \\
\text { rUrGrA rArUrA rCrArA rAdTdT-3' }\end{array}$ \\
\hline $\mathrm{DNA}_{12}$-sense strand & $\begin{array}{l}5^{\prime}-d T d C d T \text { dGdCdC dTdCdC dAdCdT rGrCrU } \\
\text { rArUrG rGrGrC rUrGrA rArUrA rCrArA } \\
\text { rAdTdT-3' }\end{array}$ \\
\hline $\mathrm{DNA}_{18}$-sense strand & $\begin{array}{l}5^{\prime}-d C d C d T \text { dAdAdC } d T d C d T \text { dGdCdC } d T d C d C \\
\text { dAdCdT rGrCrU rArUrG rGrGrC rUrGrA } \\
\text { rArUrA rCrArA rAdTdT-3' }\end{array}$ \\
\hline
\end{tabular}

Note: The RED part in the sense strands is complementary to RCA products from the GREEN part in RCA Template 


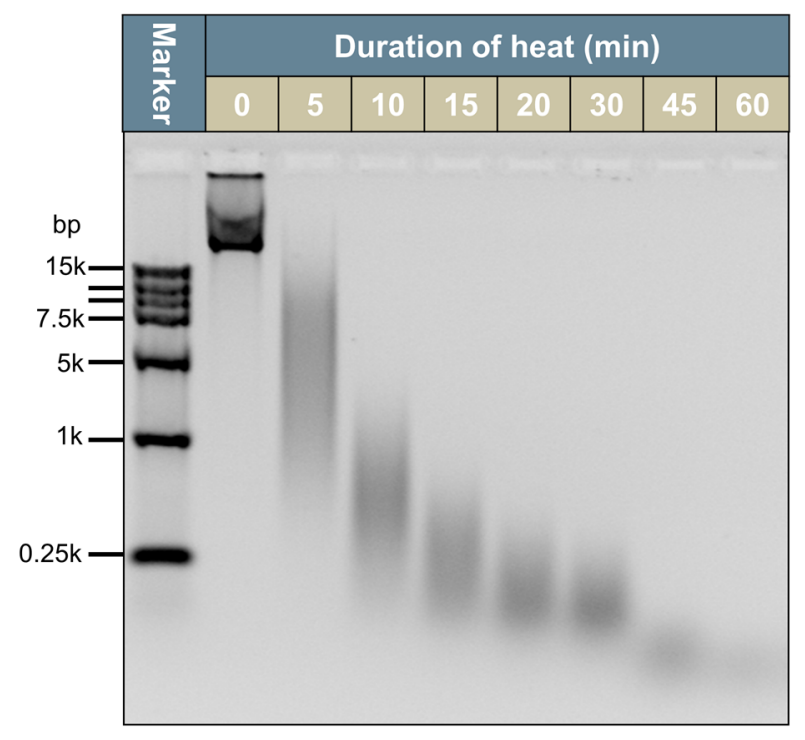

Figure S1. The agarose gel electrophoresis analysis of thermal cleaved RCA chains by heating for different minutes. 


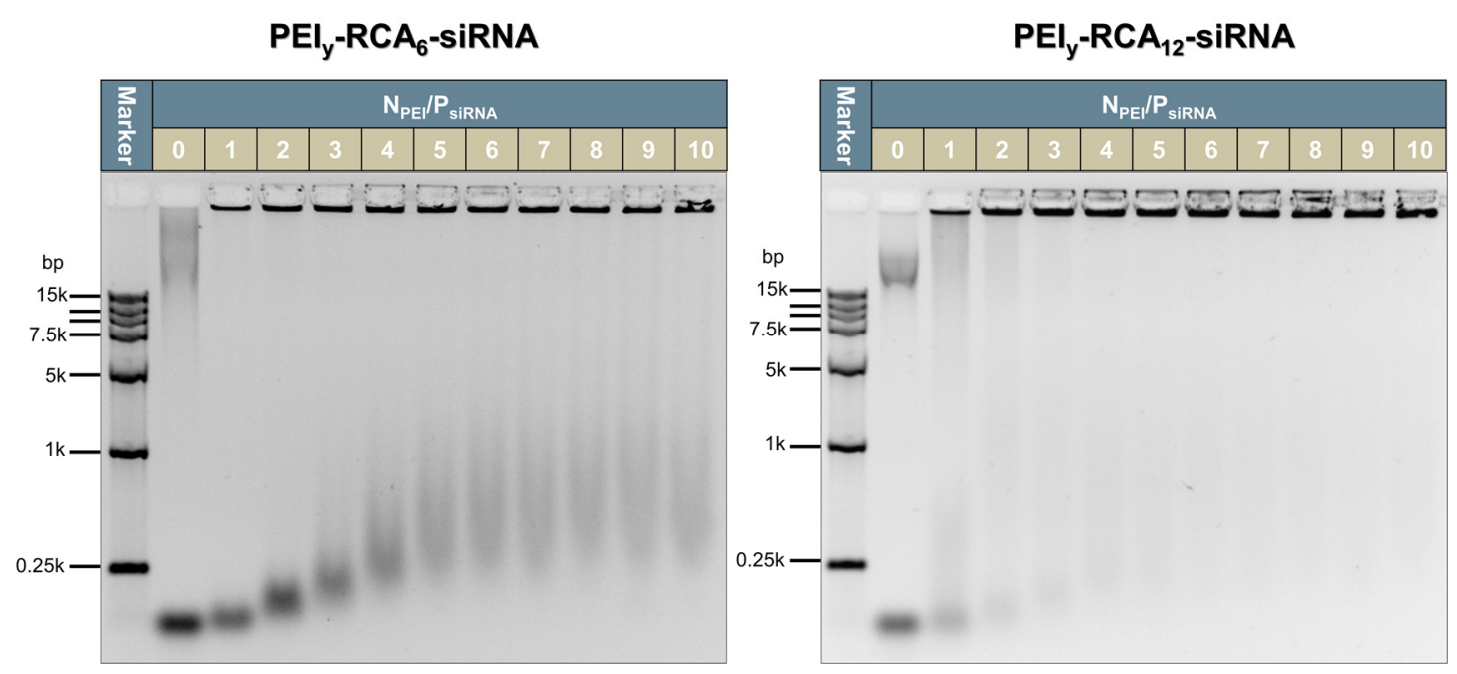

Figure S2. The agarose gel electrophoresis analysis of the complexation of the RCA6siRNA and RCA12-siRNA in the presence of PEI. 


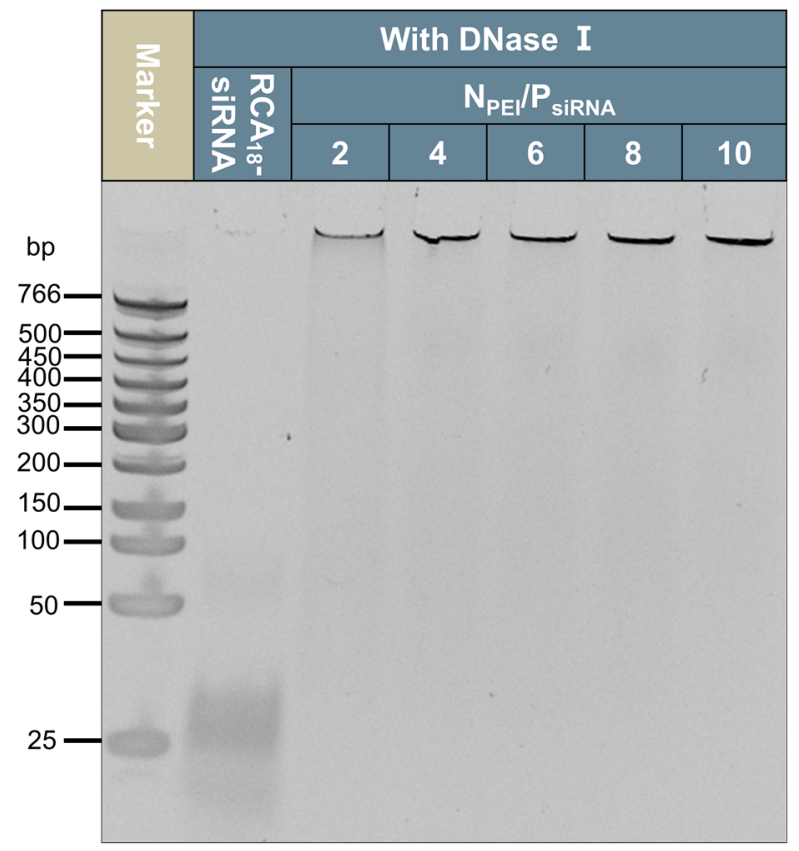

Figure S3. Polyacrylamide gel electrophoresis analysis of $\mathrm{PEI}_{\mathrm{y}}-\mathrm{RCA}_{18}-\mathrm{siRNA}$ incubated with DNase I for 30 min. 


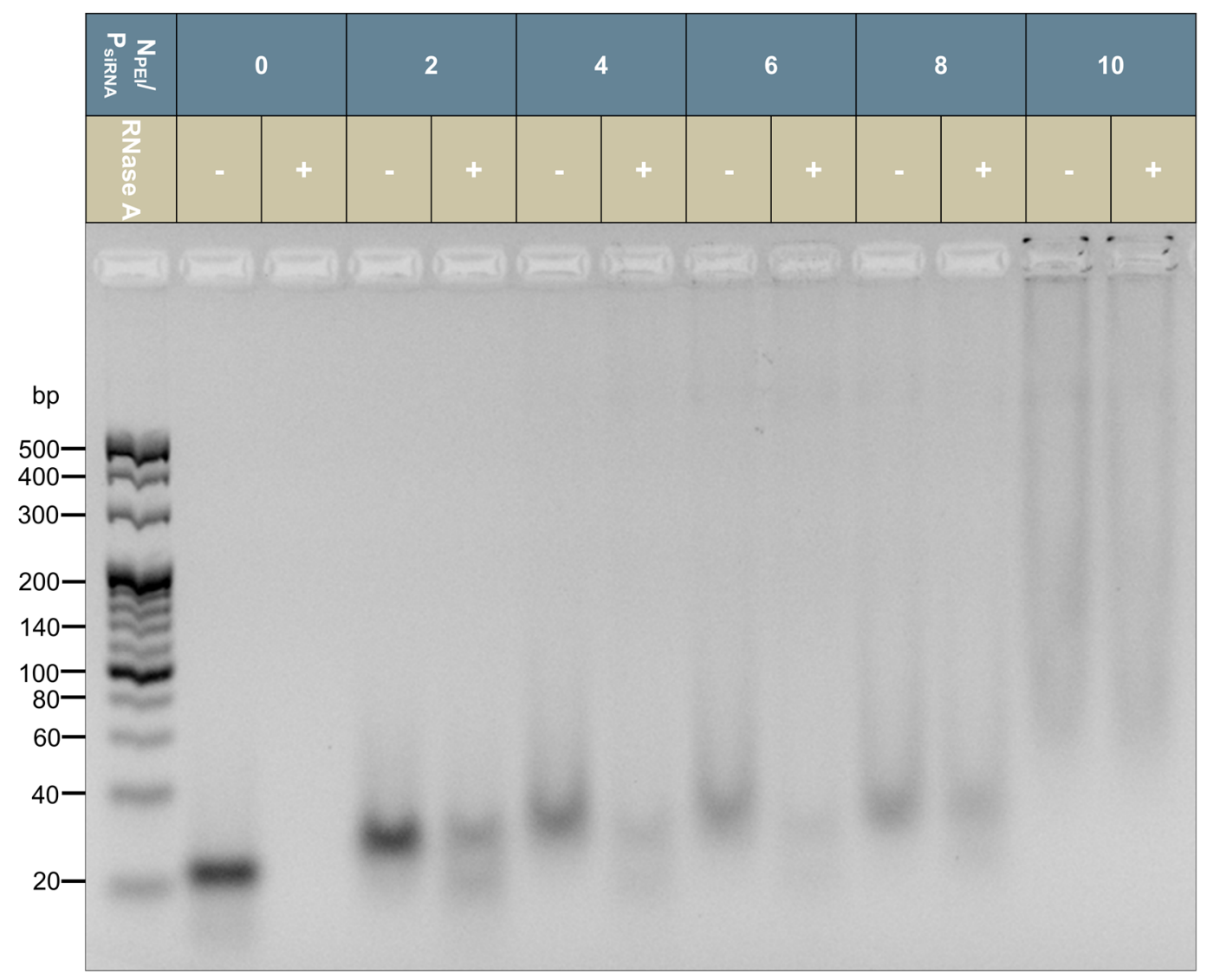

Figure S4. Stability of PEIy-siRNA incubated with/without RNase A for $30 \mathrm{~min}$. 


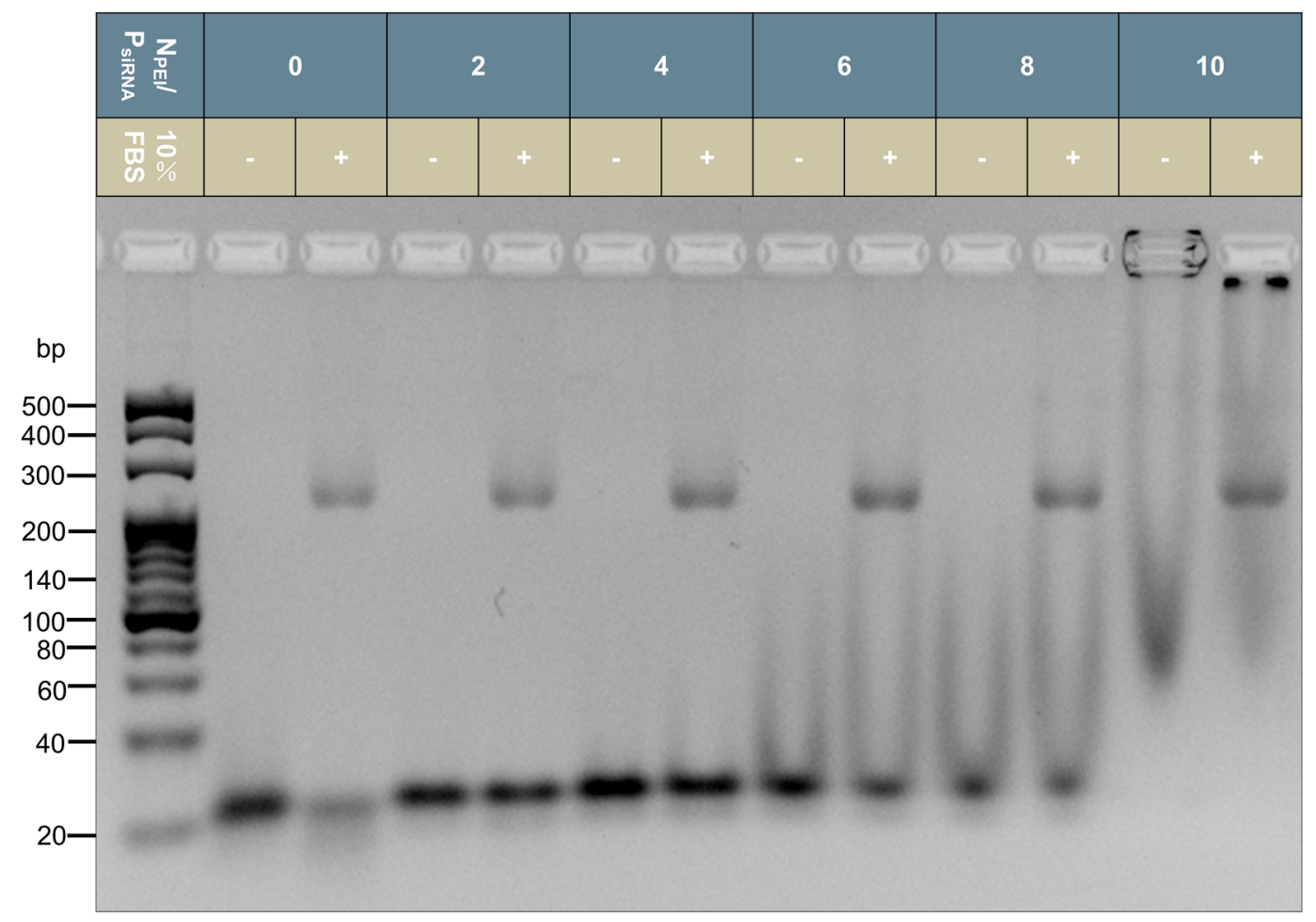

Figure S5. Stability of PEI $\mathrm{P}_{\mathrm{y}}$-siRNA incubated with/without $10 \%$ of FBS for $12 \mathrm{~h}$. 


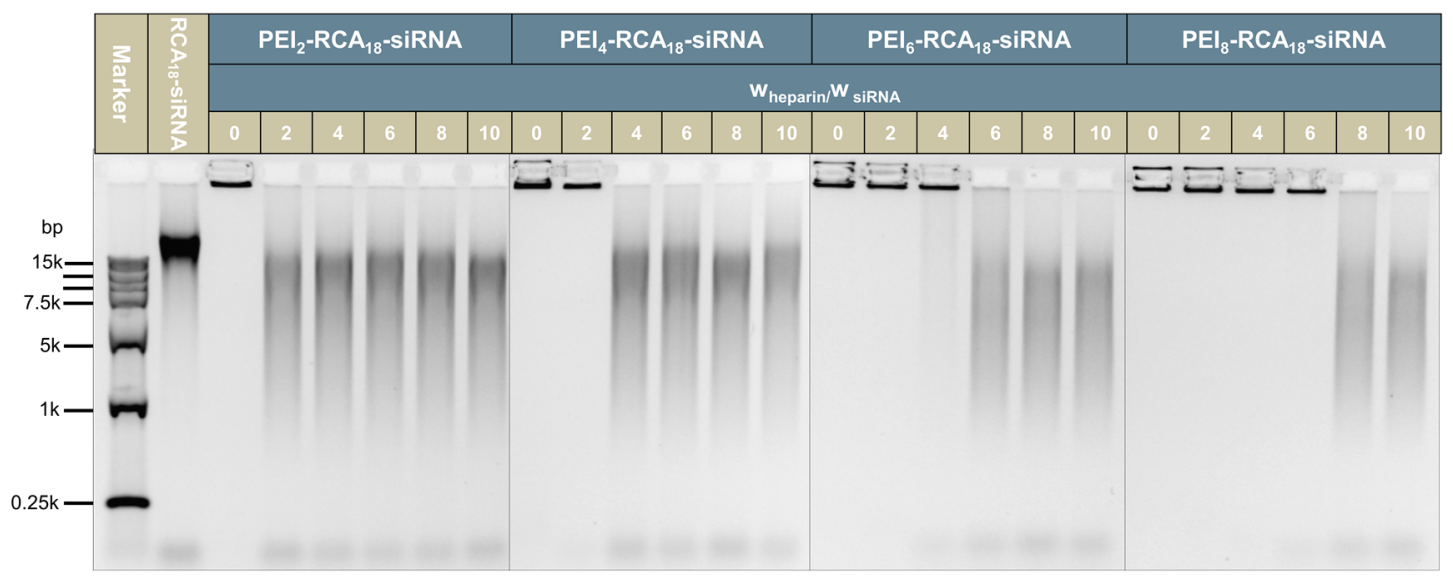

Figure S6. The dissociation property of $\mathrm{PEI}_{\mathrm{y}}-\mathrm{RCA}_{18}$-siRNA under the presence of heparin. When the NPEI/PsiRnA ratio was $2,4,6,8$, different amounts of heparin was used to incubate with the polyplex for $30 \mathrm{~min}$. 


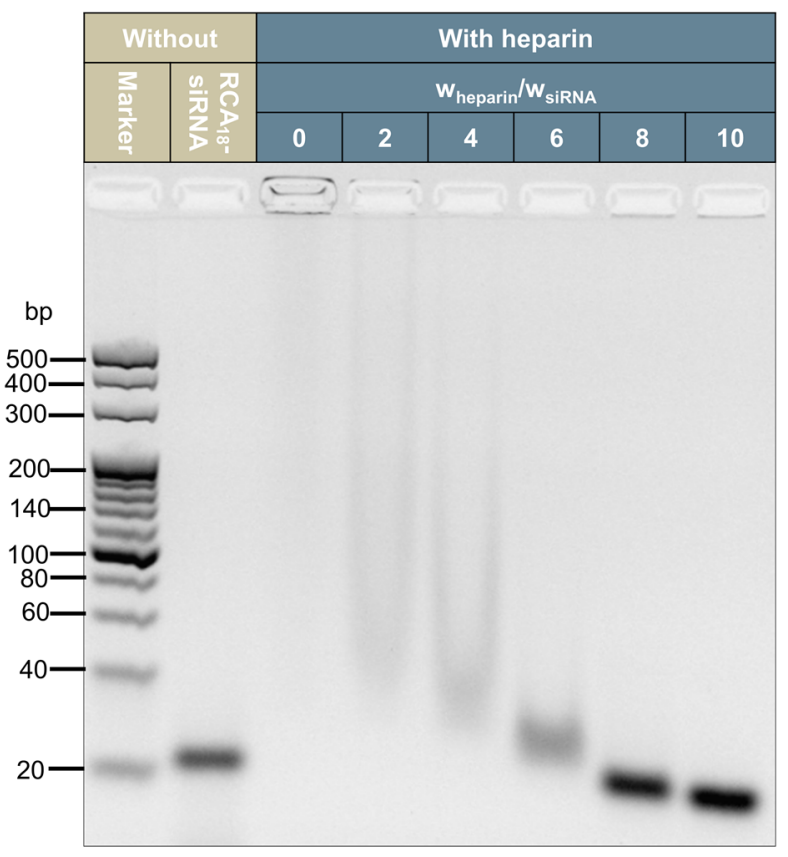

Figure S7. The dissociation property of PEI10-siRNA under the presence of heparin. 


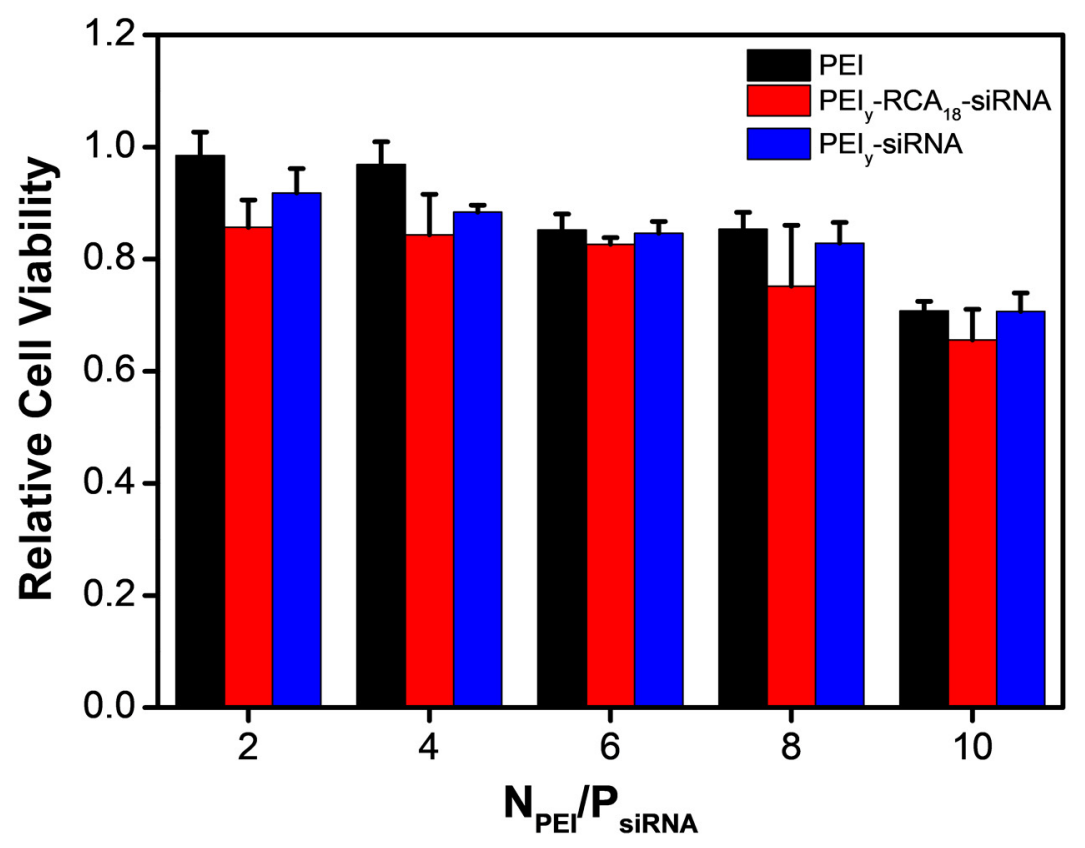

Figure S8. Cell viability of A549-luc cells incubated with of two kinds of polyplexes, PEI $y$-siRNA and $\mathrm{PEI}_{\mathrm{y}}-\mathrm{RCA}_{18}$-siRNA (siRNA amount equivalent to 5 pmol for all the polyplexes) after a 12-h incubation. The cytotoxicity of PEI at the same concentration as that in the polyplexes was tested as a reference. 